Съсоев М. С. Религиозный опыт и свидетельство // Философия. Журнал Высшей школы экономики. - 2020. - Т. 4, № 4. - С. 50-76.

\title{
МатвеЙ СЫСоев*
}

\section{РЕЛИГИОЗНЫЙ ОПЫТ И СВИАЕТЕЛЬСТВО**}

Получено: 28.01.2020. Рецензировано: 20.04.2020. Принято: 12.10.2020.

Аннотация: В статье рассматривается проблема передачи религиозного опыта, состоящая из двух элементов: проблемы особенностей индивидуального религиозного опыта и проблемы свидетельства в отношении религиозного опыта. Религиозный опыт, как кажется, сложнее поддаётся передаче посредством свидетельства, чем иные виды опыта. Поиск причин возникновения данных особенностей приводит либо к утверждению, что это проявление особенностей самого религиозного опыта, либо к утверждению, что это проявление особенностей определенного вида свидетельства. Исходя из этих особенностей и чаще всего принимая первый вариант, противники теизма пытаются редуцировать религиозный опыт к когнитивным искажениям и нарушениям, а сторонники теизма-создать особое эпистемологическое описание данного опыта с целью его защиты. Автором предпринята попытка обоснования тезиса, что трудности в передаче религиозного опыта возникают в специфических случаях и только вследствие особенностей определенного вида свидетельства, а не вследствие особенностей религиозного опыта как такового. Указанные утверждения базируются на предложенном автором подходе к анализу объектов религиозного, научного и обыденного опыта. Аанный подход объединяет естественные и сверхъестественные объекты опыта в единый класс объектов. Наиболее значимой особенностью подхода является утверждение, что такие уникальные сверхъестественные объекты опыта, как чудеса, отличаются от иных объектов опыта только эпистемически, но не онтологически. Рассматривая эпистемологические следствия данного подхода, автор приходит к заявленным тезисам.

Ключевые слова: эпистемология религии, теизм, религиозный опыт, сверхъестественное, свидетельство, передача знания.

DOI: $10.17323 / 2587-8719-2020-4-50-76$.

Получение, обработка и передача знания - три ключевых элемента познавательной деятельности. Подавляющее большинство имеющихся у нас знаний мы получаем на основании чужих свидетельств, поэтому передача знания количественно преобладает (Суинберн, Кедрова, 2014: 419). Проблема свидетельства невероятно важна, что находит отражение в эпистемологии. Однако, проследив первоначальный источник возникновения знания, которое мы получили на основании свидетельства, мы обнаружим, что им является опыт какого-либо субъекта. В связи

* Сысоев Матвей Сергеевич, ассистент кафедры философии и гуманитарной подготовки, Воронежский государственный медицинский университет им. Н. Н. Бурденко (Воронеж), sysoev.paritet@gmail.com, ORCID: 0000-0003-1152-577X.

** (C) Сысоев, М. С. (C) Философия. Журнал Высшей школы экономики. 
с этим полезно рассмотреть проблему свидетельства в совокупности с исследованием особенностей получения опыта. Наиболее актуальной в данном направлении исследований является, на мой взгляд, проблема передачи религиозного опыта (ППРО). Религиозный опыт, несомненно, обладает определенной спецификой, однако существуют различные мнения относительно того, насколько значительно его отличие от обыденного и научного опыта ${ }^{1}$ и, главное, каким образом это влияет на познавательную деятельность и на её результат в виде знания. Для упрощения рассуждений в данной статье я буду использовать словосочетание «религиозное знание», понимая под этим знание, которое изначально сформировано на основании религиозного опыта. Такое знание по своей сути является истинным и обоснованным убеждением в том, что обстоятельства, представленные в религиозном опыте, действительно имели место. Основной вопрос, который здесь можно задать, звучит так: каким образом особенности приобретения религиозного опыта влияют на его передачу? Однако я бы хотел рассмотреть эту взаимосвязь в обратном порядке и проследить, каким образом передача опыта влияет на наше представление об особенностях религиозного опыта. В ходе этого рассмотрения я постараюсь ответить на вопрос: чем обусловлена проблема передачи религиозного опыта: внутренними особенностями самого опыта или особенностями передачи опыта?

Опыт можно назвать субъективным тогда, когда его невозможно передать или он принципиально не может быть получен другим субъектом². Далее я буду использовать термин субъективность применительно к религиозному опыту, разделяя её на два подтипа. Первый случай, т. е. невозможность передачи, уместно назвать слабой субъективностью. Слабо субъективный опыт - это такой опыт, который по тем или иным причинам не может быть передан. Второй случай, т. е. принципиальную невозможность приобретения опыта другим субъектом, можно назвать сильной субъективностью. Опыт со свойством сильной субъективности - это такой опыт, который полностью принадлежит к ментальной

${ }^{1}$ Далее я буду использовать обобщенный термин - нерелигиозный опыт.

${ }^{2}$ Под «передачей» в данном случае понимаются все возможные виды свидетельства, подробнее о которых будет сказано во втором разделе. Под «получением» опыта понимаются все возможные виды получения опыта, включая получение индивидуального опыта через его переживание, а также получение опыта в результате передачи опыта. Поэтому для целей данной статьи можно считать, что второй случай субъективности включает в себя первый. 
жизни определенного субъекта. В самом очевидном случае- - это субъективность невыразимых качественных характеристик опыта, а также чувство, «каково это быть» определенным субъектом. При этом, если мы выделяем несколько типов свидетельства, то вполне можно предположить, что субъективность будет распространяться не на все типы свидетельства. В этом случае мы должны будем локализовать субъективность опыта в одном из видов свидетельства, т. е. обозначить пределы субъективности.

Утверждения, которые я хотел бы обосновать в рамках представленного далее подхода, состоят в следующем:

(А) Религиозный опыт субъективен в слабой форме.

(в) Слабая субъективность религиозного опыта локализована в одной из разновидностей свидетельства.

(с) Слабая субъективность религиозного опыта не связана с особенностями приобретения религиозного опыта и не является его внутренним свойством, а обусловлена только особенностями одной из разновидностей свидетельства.

Для дальнейших рассуждений имеет значение различие между двумя подходами к анализу передачи опыта, каждому из которых будет соответствовать своя особенная версия проблемы передачи опыта. Вопервых, опыт может быть передан в форме предоставления свидетельских данных. Элементы опыта иногда именуются данными (evidence) и в эвиденциалистском подходе служат для обоснования убеждений. Поэтому свидетельство о полученном опыте иногда называют свидетельскими данными (testimonial evidence) ${ }^{3}$. Во-вторых, опыт может быть передан путем передачи знания, сформированного на основании этого опыта. Для этого конструкцию нужно усложнить и установить, что сначала полученный опыт обосновывает убеждение субъекта. Если обоснованное убеждение является истинным, то, согласно критериям $\mathrm{JTB}^{4}$, оно является знанием. Затем субъект передаёт полученное знание путём свидетельствования. Точка зрения, что свидетельство может являться основанием для возникновения или способом передачи знания,

3Пример использования такого словосочетания см. Rowley, 2012. Однако понимание этого словосочетания в данной статье будет несколько отличаться в связи с особенностями моего подхода.

4JTB (justified true belief) - истинное обоснованное убеждение. Несмотря на критику Э. Геттиера, я не считаю эти критерии неприменимыми. На мой взгляд, большую часть ответов на критику Геттиера следует рассматривать не как новые подходы к пониманию знания, а, скорее, как уточнение критерия обоснованности. 
достаточно распространена, но не является общепризнанной (Adler, 2006). В случае когда содержание убеждения, сформированного на основании опыта, представляет из себя только отчет об этом опыте, передачу знания можно считать усложненной версией передачи опыта. Существуют смешанные или промежуточные подходы к анализу передачи опыта, однако я не буду подробно останавливаться на них. Рассуждения в отношении проблемы передачи опыта будут касаться в первую очередь проблемы передачи знания на основании этого опыта, поскольку это более специфическая версия рассматриваемой проблемы. Однако все рассуждения будут также справедливы в отношении передачи свидетельских данных, поскольку для этого требуется выполнение значительно меньшего количества условий.

Я начну с того, что изложу свой взгляд на соотношение объектов религиозного и нерелигиозного опыта, поскольку от этого в первую очередь зависит понимание религиозного опыта. Затем я перейду непосредственно к вопросу о передаче опыта и представлю обоснование тезисов (в), (с). В завершение я вернусь к тезису (А) и представлю обоснование утверждения о слабой субъективности религиозного опыта, поскольку для этого мне потребуется предшествующая аргументация. В ходе повествования я попробую показать, что изложенный мной подход к рассмотрению объектов опыта обладает рядом преимуществ при объяснении проблемы передачи религиозного опыта. Они заключаются в том, что данный подход помогает обосновать вышеуказанные тезисы и объяснить проблемы, возникающие при передаче религиозного опыта, не прибегая к установлению различий между религиозным и нерелигиозным опытом. Все различия оказываются незначительными и возникают лишь тогда, когда религиозный опыт совмещается с достаточно редко используемой разновидностью свидетельства. Необходимо сделать оговорку, что я не буду рассматривать вопрос о существовании Бога, чудес и приму их существование в качестве удобной для работы аксиомы. Рассуждения, представленные в данной работе,-это общие штрихи на пути построения универсальной концепции, однако в рамках моего собственного мировоззрения они полностью реализуют вышеуказанные преимущества.

\section{ОБЪЕКТЫ РЕЛИГИОЗНОГО И НЕРЕЛИГИОЗНОГО ОПЫТА}

Объект, который является источником опыта, отличает религиозный опыт от нерелигиозного, поэтому я начну с рассмотрения объектов, специфических для религиозного опыта. Уильям Джеймс в работе 
«Многообразие религиозного опыта» указывает следующее (Джеймс, Малахиева-Мирович и Шик, 2017: 24):

Так как всякое конкретное душевное состояние может быть разложено на ощущение плюс специфический объект, которым последнее вызвано, то и религиозное чувство, как психическое явление, может быть выделено из среды всех других конкретных чувствований.

Исходя из этого критерия, объектами, вызывающими религиозный опыт, если мы предполагаем его достоверность, являются некие специфические явления. И это не только собственно объекты, но и события 5 . Основным объектом, вызывающим религиозный опыт, в таком случае будет Бог, по крайней мере, когда речь идет о монотеистических религиях. Я остановлюсь на следующей позиции: Бог может восприниматься либо через сверхъестественное явление, либо через естественное. Вопрос о том, возможно ли непосредственное восприятие сверхъестественного Бога, является крайне сложным, и я собираюсь оставить его открытым, поскольку его рассмотрение увело бы дискуссию в сторону ${ }^{6}$ В данном случае меня будет интересовать не сам Бог, который действует, образуя некое явление, а множество явлений религиозного опыта, которые чем-то отличаются от естественных явлений, но при этом не являются Богом. Обычно именно такие явления называют сверхъестественными. Объектами, вызывающими нерелигиозный опыт, являются естественные объекты. Существуют две наиболее распространенные точки зрения на соотношение сверхъестественных и естественных явлений, которые мне нужно рассмотреть, прежде чем перейти к моей собственной.

Традиционной является мягкая форма противопоставления сверхъестественных и естественных явлений. В качестве примера можно назвать подход Фомы Аквинского, который указывает, что

нечто трудное называют чудесным не потому, что оно превосходит то, в чем оно сделано, но потому, что оно превосходит возможности природы, равно

5В этой связи следует различать два понятия: объект как нечто, являющееся источником опыта, и объект как некая вещь. В словосочетании «объект опыта» понятие объект используется в первом смысле.

${ }^{6}$ Уклонение от этого вопроса я считаю допустимым, потому что - на мой взглядотсутствует убедительное обоснование тезиса о возможности непосредственного восприятия сверхъестественного Бога. А поскольку в рассуждениях я следую от (очевидных для меня) естественных объектов к сверхъестественным, а не наоборот, то обоснованию «по умолчанию» подлежит именно тезис о возможности непосредственного восприятия сверхъестественного, а не противоположный тезис. 
как и необычным называют не [просто] редкое, но происходящее вне обычного естественного порядка вещей (Фома Аквинский, Еремеев, 2005: 400).

Этот подход порождает группу сверхъестественных явлений, качественно отличающихся от естественных. Есть некий естественный порядок, в соответствии с которым обычно всё происходит, а есть превышающее его сверхъестественное, которое происходит вне этого порядка. Отсюда следуют вопросы о различии между естественным и сверхъестественным, о нашем опытном доступе к сверхъестественным явлениям и все последующие различия в религиозном и нерелигиозном опыте. Даже при наличии удовлетворительных ответов на эти вопросы такой подход значительно усложняет систему нашего восприятия этих явлений.

В Новое время получает распространение подход, который более жестко противопоставляет сверхъестественные явления естественным с целью продемонстрировать недостоверность опыта наблюдения сверхъестественных явлений. Дэвид Юм определяет чудо как «нарушение законов природы», и при этом «всякому чудесному явлению должен быть противопоставлен единообразный опыт, иначе это явление не заслуживает подобного названия» (Hume, 2007: 100). Любые подходы, рассматривающие сверхъестественное как нарушения законов природы, содержат в себе внутренние противоречия. Закон природы-в отличие от закона, созданного людьми, - не может быть нарушен по определению в рамках той системы, в которой он действует, в противном случае он не может быть назван законом. Если же ссылаться на то, что Бог сам является создателем этой системы и не подчиняется данным законам, то в каком смысле он может нарушать данные законы? Например, Ульрих Геккель и Петр Покорны пишут о Новозаветных чудесах усмирения бури и хождения по воде следующее: «...речь не идет о том, что Иисус нарушает законы природы, Он властвует над стихиями, как Творец» (Покорны и Геккель, Витковский, 2019: 385). Рассуждения Юма, конечно, находятся в несколько другой плоскости, поскольку он прямо утверждает, что под законами природы он понимает не фундаментальное свойство вселенной, а зафиксированную в опыте регулярность повторения явлений. Это обнаруживает сразу два слабых места в его подходе. Во-первых, такой закон непрерывно меняется под воздействием опыта, и поэтому он не является чем-то устойчивым и надежным. Во-вторых, Юм сводит оценку чудес к явлениям, которые до этого никогда не наблюдались, говоря, что нечто 
будет чудом, если «такое явление не наблюдалось никогда, ни в одну эпоху и ни в одной стране» (Hume, 2007: 101). Эта точка зрения заранее исключает возможность адекватного познания не только уникальных событий, т. е. таких событий, которые могут произойти только один раз, но и прибавляет к списку уникальных событий еще и редкие мнимо-уникальные события, т. е. такие, которые происходили редко, но в опыте, доступном человеку, появлялись всего лишь единожды. Это другая крайность в анализе сверхъестественных событий, которая не позволяет их учитывать в нашем опыте адекватным образом.

В вышеприведенных отрывках упоминаются чудеса, которые обычно относятся к сверхъестественным явлениям. Однако это не тождественные понятия: по крайней мере, есть такие сверхъестественные явления, которые с трудом можно назвать чудесами. Хотя меня интересует весь диапазон сверхъестественных явлений, а не какие-то их конкретные разновидности, отличия чудес от сверхъестественных явлений необходимо кратко рассмотреть, поскольку оно играет большую роль. Далее я сведу все разновидности сверхъестественных явлений к двум типам: уникальные (чудеса) и повторяющиеся. Во-первых, смысл слова «чудо» связан с определенным субъективным аспектом-с удивлением, а уже потом происходит переход к объективному значению (Покорны и Геккель, Витковский, 2019: 379). В Новом Завете чудеса-это проявления божественной власти и могущества, которые доказывают, предвещают, знаменуют, удивляют (не в праздном смысле, а с целью ввести наблюдателя в особое состояние благоговения). Во-вторых, это уникальные события. В отличие от чудес иные сверхъестественные явления могут повторяться достаточно часто, однако всё еще быть сверхъестественными или, как это формулирует Фома, превышающими естественный порядок. Пример уникального сверхъестественного явления - это хождение Христа по воде. Предположим, что у нас есть два свидетеля чуда: сторонник Юма (Ю) и сторонник Фомы $(\Phi)$. Они наблюдают хождение по воде, которое, согласно их опыту и познаниям, невозможно. Ю, вероятно, не поверит своим глазам, если это произойдет всего лишь раз. Однако он может пояснить, что если это повторится, то он, вероятно, изменит свои представления о физических свойствах воды. Кроме того, Ю уж точно не поверит свидетельству о подобном событии, ибо «никакое свидетельство недостаточно для установления чуда, кроме такого, ложность которого была бы большим чудом, нежели тот факт, который оно стремится установить» (Hume, 2007: 101). Ф, вероятно, увидит в самом факте противоречия этого события опыту проявление 
его сверхъестественности и решит, что это вмешательство сверхъестественного в естественный порядок. Из этих особенностей следует, что причины, по которым чудо можно назвать сверхъестественным, очевидны каждому, кто может его наблюдать, поскольку опыт восприятия чуда отличается от опыта восприятия других явлений.

Пример повторяющегося сверхъестественного явления - превращение хлеба в тело Христово (пресуществление) в процессе таинства Евхаристии. Наиболее распространенная интерпретация того, что при этом происходит, следующая: произошло замещение субстанции хлеба на тело Христово, хотя акциденции хлеба и восприятие его как хлеба сохраняются (Прусс, Васильев, 2013: 762). Ю в этом случае скажет, что ничего не произошло, ведь восприятие хлеба не изменилось. Чувственное восприятие хлеба будет у обоих свидетелей чуда идентично, но представления о субстанции объекта будут различаться. Однако сторонник Фомы будет утверждать, что хлеб преобразился, не на основании собственного восприятия, а на основании свидетельства. Цепочка таких свидетельств уходит в прошлое к авторитету Откровения. Поэтому если рассматривать опыт, вызванный непосредственно объектом, то религиозный и нерелигиозный опыты не будут отличаться.

Между первым и вторым случаями есть принципиальное отличие, которое заключается в том, как полученный опыт встраивается в структуру воззрений субъекта $\Phi$ и субъекта Ю. Восприятие чуда в первом случае само по себе не будет отличаться для $\Phi$ и Ю, и, на первый взгляд, отличаться здесь будет лишь оценка опыта данными субъектами. Однако, если мы рассмотрим опыт в контексте всей совокупности опыта $\Phi$ и Ю (а только так этот опыт и может существовать), то опыт $\Phi$ и опыт Ю противоречат друг другу, поскольку для $\Phi$ это было чудо, а для Ю-галлюцинация, мираж или заблуждение вследствие недостаточности данных. Установка Ю, как было указано выше, предписывает ему не доверять однократно произошедшим событиям, поэтому Ю будет анализировать случившееся особым образом, возможно, даже утверждая, что «на самом деле» его не было. Рассмотрим их предполагаемый отчет о восприятии. Ю, скорее всего, скажет: «На самом деле я не воспринимал этого». Именно так ведут себя люди, уверенные в том, что столкнулись с иллюзией. При этом $\Phi$, наоборот, отметит, что он «воспринимал» и воспринимал нечто подлинное, даже если он не готов сразу признать, что это было чудо. При этом у $Ф$ и Ю есть конкретная причина для расхождения во мнениях - различие в оценке уникальных событий. Во втором случае ситуация совершенно иная и столь 
очевидных противоречий не возникает. Исходя из вышеуказанной интерпретации преосуществления, $\Phi$ воспринимает вкус и свойства хлеба и Ю воспринимает вкус и свойства хлеба, отчет о восприятии у Ю и Ф не будет отличаться ни сам по себе, ни в контексте всего их жизненного опыта. Разумеется, в совокупности всех сопутствующих восприятию переживаний $\Phi$ будет испытывать совершенно иные чувства и опыт: например, вкушение хлеба у него будет совершенно иным, но здесь мы четко видим, в чем различие: оно заключается не в опыте, вызванном самим объектом, а в принятии $\Phi$ свидетельства Церкви. Опыт $\Phi$ формируется из двух компонентов: собственного опыта и свидетельства. И-в отличие от первого типа сверхъестественного явления - среди людей никогда не существовал такой субъект, который обладал бы этим знанием не на основании свидетельства7. Это означает, что проблема передачи религиозного опыта, вызванного сверхъестественным явлением второго типа, никак не может быть связана с особенностями личного опыта какого-либо субъекта. Отсюда следует, что в отношении второго типа сверхъестественных явлений заявленный в начале статьи тезис (с) уже обоснован. Поэтому далее мои рассуждения в этом разделе будут касаться только очевидных сверхъестественных явлений, которыми являются чудеса, поскольку именно они вызывают противоречие в опыте, и поэтому особенности передачи такого опыта предположительно могут отличаться из-за особенностей опыта. К сверхъестественным явлениям второго типа я обращусь в последнем разделе статьи при обосновании тезиса (А).

Вернёмся к противоречию между отчетами $\Phi$ и Ю в случае опыта восприятия чуда. Оба подхода базируются на определенных фундаментальных установках, которые и вызывают противоречие. В мои задачи не входит критика этих фундаментальных установок - я лишь хочу предложить описание своей точки зрения, которая одинаково допускает существование сверхъестественного Бога, и при этом не исключает естественную интерпретацию всех явлений, и устраняет противоречия в опыте. Альтернатива этим подходам заключается в том, чтобы считать все объекты, вызывающие опыт, естественными, пока у нас нет оснований для выделения отдельной категории объектов. Предлагая называть

7 В данном случае я не учитываю Христа, который мог бы являться таким первоначальным субъектом, поскольку его опыт, очевидно, должен отличаться от привычного нам. Поскольку я не рассматриваю вопрос о достоверности этого свидетельства, то нет необходимости углубляться в теологические аспекты этого вопроса. 
объекты естественными «по умолчанию», я исхожу из предположения, что существует как минимум один сверхъестественный объект-Бог, однако может ли Бог быть сам по себе объектом опыта непосредственно неизвестно. Отличие от точки зрения Фомы заключается в том, что существование иных сверхъестественных явления не предполагается заранее. Отличие от точки зрения Юма заключается в том, что сверхъестественные явления (а точнее то, что именуется «сверхъестественным» в подходе Фомы) не отрицаются и являются такими же полноправными объектами опыта, как и другие естественные явления.

Первым значимым компонентом данного подхода является позиция Готфрида Вильгельма Лейбница в отношении сверхъестественных и естественных явлений, а также некоторые утверждения Самюэля Кларка, высказанные им в переписке с Лейбницем. Лейбниц пишет в работе «Рассуждения о Метафизике» (Лейбниц, Бобров, 1982: 141):

...если мы понимаем в нашей природе все, что она выражает, то ничто не бывает для нее сверхъестественным, так как она простирается на все, ибо действие всегда выражает свою причину, а Бог есть истинная причина всех субстанций.

Здесь отмечается первый важный аспект моего подхода: если Бог существует, то он является единственной причиной всех явлений. В переписке с Лейбницем Самюэль Кларк отмечает, что различие между естественным и сверхъестественным, очевидно, существует только для нас, но не онтологически.

Придать Солнцу (или Земле) регулярное движение-это мы называем «естественным»; остановить же его на один день - это мы называем «сверхъестественным», но оно не требует большей мощи, чем другое; а для Бога и то и другое в равной мере естественно и сверхъестественно (Лейбниц и Кларк, Свидерский и Кребер, 1982: 440).

В одном из последующих писем Кларк высказывает точку зрения, что если мы не считаем, что у Бога существует два типа действий разной степени могущества, то у нас есть только две альтернативы (там же: 524 ):

...различение между естественными и сверхъестественными действиями Бога является чем-то имеющим значение только для нас, поскольку мы называем обычное проявление могущества Бога «естественным», а необычное «сверхвестественным». 
...в одном случае (сверхъестественное) Бог действует непосредственно, а в другом (естественное) его деятельность опосредуется вторичными причинами (Лейбниц и Кларк, Свидерский и Кребер, 1982: 524).

Первая альтернатива предполагает, что различие между естественным и сверхъестественным лишь эпистемическое, но не онтологическое. Причем критерием сверхъестественности является необычность действия. K этому подходу я вернусь далее. Согласно второй альтернативе различие между естественным и сверхъестественным заключается не в действиях Бога, а в цепочке каузальных связей. Если Бог воздействует на что-то непосредственно, то это сверхъестественное действие, а если он воздействует опосредованно (т. е. воздействует на некоторый объект, который затем по цепочке передаёт воздействие дальше), то речь идет о естественном. Даже в этом случае есть все основания предполагать, что любые явления, встречающиеся в нашем опыте, естественны, поскольку мы наблюдаем само явление (опосредующий элемент в каузальной цепочке), которое всегда опосредует деятельность Бога. Смысл ввести категорию сверхъестественных явлений возникнет тогда, когда мы сможем обосновать, что Бог воздействует на нас непосредственно. И даже в этом случае онтологически они [явления] будут естественны, а их отличие от других явлений будет обусловлено лишь количеством звеньев в каузальной цепочке. На мой взгляд, мы не должны вводить различия в природе явлений без достаточных оснований, однако ни одна из альтернатив не приводит нас к такому различию.

Второй значимый компонент моего подхода заключается в уточнении двух вариантов понимания сверхъестественного, предложенных Кларком. Я свожу их к следующим двум альтернативам:

(I) Никакие события в мире не происходят сверхъестественно в том смысле, что Бог действует непосредственно. Любые изменения в мире связаны с использованием Богом созданных им же законов. При этом так называемые естественные события - это результат работы естественных законов, а так называемые сверхъестественные события - это результат специфического сочетания законов природы, приводящего к необычным для опыта результатам.

(II) Любые события в мире происходят сверхъестественно в том смысле, что все они являются действиями Бога. При этом так называемые естественные явления - это то, каким образом Бог обычно управляет миром, а так называемые сверхъестественные - это результаты более редких действий Бога. 
Первый вариант признаёт существование законов природы, которые созданы Богом и функционируют по заданной программе, а второй признаёт, что всё управляется Богом непосредственно и регулярно. Мне кажется более предпочтительным второй вариант, однако выбор между ними для дальнейших рассуждений непринципиален. В любом случае, согласно данному подходу, онтологически не существует различий между тем, что мы называем естественным и сверхъестественным.

Ни один из этих вариантов не связан с редукцией сверхъестественных явлений к естественным (или наоборот в случае второго подхода), как может показаться на первый взгляд. В этом смысле они симметричны, и мы не только сближаем сверхъестественное с естественным, но и естественное - со сверхъестественным. Ведь в теизме естественное не существует независимо от Бога (если речь не идет о деизме), наоборот, Бог постоянно поддерживает ${ }^{8}$ существование естественного (даже в модели (I), где существуют независимые законы). В рамках своего подхода я понимаю под естественным всё, что является результатом божественного действия прямо или косвенно. Самюэль Кларк определяет естественный порядок следующим образом (Clarke, 1998: 149):

...воля Бога, производящая определённые эффекты непрерывно, постоянно, неизменно и однородно; такой порядок или способ действий, будучи в каждом моменте совершенно произвольным, легко может быть как изменен в любой момент, так и сохранен.

Это нисколько не ослабляет обосновывающую силу происходящих необычных событий и не опровергает теистическую позицию. Сам факт того, что такие события происходят именно тогда, когда они происходят, требует объяснения. Специальное действие Бога может быть реализовано как посредством вмешательства в работу законов природы, так и посредством их использования (Суинберн, Кедрова, 2014: 366).

В качестве третьего значимого компонента моего подхода служит позиция Аврелия Августина в отношении метафизической природы чудес, которая во многом схожа с позицией Кларка в отношении сверхъестественных явлений вообще, однако Августин четче проясняет причины их кажущегося различия: он говорит о чудесах, что «на самом деле они не противны природе». Августин считает, что не «может быть противным природе то, что совершается по воле Божией», поскольку «воля Творца

${ }^{8}$ Большую роль в данных рассуждениях играет то, каким образом трактуется указанная «постоянная поддержка». Я использую указанный выше подход Кларка, но возможны и другие принципиально отличные трактовки. 
есть природа всякой сотворенной вещи» (Августин, КДА, 1998: 463). Августин отмечает: то, как люди «знают [...] природу опытным путем», может быть ошибочно. И наиболее ценным является замечание, что

уже и само то, что в природе вещей всем известно, не меньше было бы удивительным и для всякого внимательного наблюдателя поразительным, если бы люди не имели обыкновения удивляться только явлениям редким (там же: 464).

Августин здесь не высказывается о том, превышают ли чудеса естественную природу, однако это и не имеет значения, поскольку я не преследую цели реконструировать его подход. Гораздо важнее для меня его рассмотрение регулярности явлений как критерия для именования явления «чудом». Чудеса могут быть представлены как редкие и уникальные естественные явления без потери их основного содержания, если под естественным понимается всё, что является результатом божественного действия прямо или косвенно. Для того чтобы понять, насколько тонка грань между чудом и обычным событием, достаточно представить, что событие, которое мы относим к чудесному, начало повторяться. В этом случае оно не только перестанет быть чудом, но и будет включено в закон природы (Суинберн, Кедрова, 2014: 368).

Теперь на основании вышесказанного мы можем сформулировать следующую позицию. Во-первых, отсутствует онтологическое различие между естественными и сверхъественными событиями (исходя из рассмотрения указанных выше альтернатив). Во-вторых, тот религиозный опыт, который вызывает чудо, обусловлен его эпистемической, но не онтологической уникальностью. Наилучшим критерием онтологической уникальности в этом смысле могут служить происходящие при этом физические процессы и реализуемые при этом физические законы. Любое чудо может быть повторено Богом в том смысле, что те же самые физические процессы, которые сопровождали чудо, могут быть повторены. Исходя из приведенных выше рассуждений, чтобы считать иначе, у нас должны быть основания. Т. е. у нас должны быть основания для того, чтобы утверждать: явление, которое мы наблюдали и которое мы назвали чудом, не может быть реализовано с помощью естественных законов. Примером подлинной онтологической уникальности может служить сотворение вселенной. По крайней мере, можно с уверенностью сказать, что для каждой вселенной есть лишь один момент сотворения.

Не лишним будет напомнить, что данные выводы касаются только уникальных сверхъестественных явлений, т. е. чудес. После того как 
мной было введено различие между уникальными и повторяющимися сверхъестественными явлениями, я сделал вывод, что тезис (с) для повторяющихся сверхъестественных явлений уже обоснован. Представленный после этого подход обосновывает тезис (с) для уникальных сверхъестественных явлений (чудес) и, таким образом, объединяет все явления опыта в единый нейтральный класс явлений (я бы предпочел именовать все явления естественными, однако это может вызвать путаницу с иными способами определения естественного).

Итак, религиозный и нерелигиозный опыт вызваны одним общим классом нейтральных объектов опыта. Следовательно, принципиальных различий в природе доступного нам религиозного и нерелигиозного опыта быть не может. Теперь необходимо прояснить подробнее, откуда возникает та уникальная особенность религиозного опыта чуда, которая и производит основной эффект на наблюдателя. В данном подходе особенностью объекта, вызывающего религиозный опыт, становится его эпистемическая уникальность и этот же фактор является причиной, по которой данные явления предстают перед нами как чудеса в ценностном смысле. Чудеса не обладают свойством онтологической уникальности, однако обладают свойством эпистемической уникальности. Т.е., не отличаясь от естественных явлений онтологически, такие сверхъестественные явления, как чудеса, отличаются от них эпистемически, а именно с точки зрения нашего восприятия. Хочу еще раз подчеркнуть, что это не устраняет религиозного содержания чуда, поскольку эпистемическая уникальность явления для определенной группы субъектов - это предполагаемая причина, по которой Бог выбирает именно её для донесения до людей своего послания. Христос ходил по воде, поскольку это могло произвести необходимый эффект удивления. А эффект удивления, в свою очередь, был вызван эпистемической уникальностью события. Поэтому выбор того, каким образом будет реализовано чудо, напрямую зависит от того, какие события являются эпистемически уникальными. Онтологическое единство уникальных и повторяющихся событий не имеет самостоятельного значения для религиозного эффекта, поскольку на восприятие события как чуда влияют эпистемические критерии. Если, например, существовали десятки случаев хождения по воде, однако все очевидцы умерли и не оставили об этом никаких свидетельств, то хождение Христа по воде произведет точно такой же эффект, поскольку в опыте наблюдающих за ним людей такого не случалось. Таким образом, опытных противоречий между религиозной и нерелигиозной точкой зрения в данном случае не возникнет. 
В связи с изложенным в настоящем разделе может возникнуть два вопроса.

Во-первых, каким образом индивидуальный субъект может отличить чудо от иного эпистемически уникального события? Ответ заключается в том, что отличить его в отрыве от остальных явлений опыта невозможно, как невозможно и понять, в каких случаях чудо является чудом, а в каких - не является. Однако, исходя из моего анализа, это и не имеет особого значения, ведь подлинная роль чуда-удивлять, т. е. производить эффект на восприятие. Оно и не должно отличаться от остальных событий онтологически, поскольку причиной любого события в данном подходе так или иначе является Бог. Сама же эта проблема не связана с моим подходом, она связана с ограниченностью знаний человека. Вера в то, что именно Бог творит чудеса как демонстрацию своего могущества именно человеку, предполагает, что человек сможет как-то отличить подлинное чудо от неподлинного. Однако следует отметить, что речь идет лишь о вере и о вероятностной оценке. Обрести окончательное и достоверное знание того, что источником какого-либо явления является Бог, невозможно по той же причине, по которой нам недоступно восприятие качественного преображения хлеба в тело Христово в процессе Евхаристии.

Во-вторых, означает ли всё вышесказанное, что эпистемическая уникальность является необходимым и достаточным свойством чуда? Мой ответ: нет. Однако, на мой взгляд, эпистемическая уникальность-это тот критерий, который влияет на признание того или иного явления чудом. Более того, я допускаю, что явления, вообще никак не связанные ни с одной религией, могут быть значительно более редкими, чем некоторые чудеса. Как я указал выше, оценить, является ли определенное явление чудом, мы можем лишь вероятностно ${ }^{9}$.

Далее я рассмотрю, каким образом представленный подход объясняет различия в передаче опыта, и завершу мысль о том, что эти различия не связаны с особенностями приобретения опыта.

\section{ПРОБЛЕМА ПЕРЕДАЧИ ЗНАНИЯ И РЕЛИГИОЗНЫЙ ОПЫТ}

Теперь я обращусь непосредственно к проблеме знания на основании свидетельства. Джон Греко формулирует обозначенную проблему следующим образом (Греко, Карпов, 2017: 25):

${ }_{9}^{9}$ Благодарю анонимных рецензентов за данные вопросы. 
...свидетельские данные, говоря эпистемологически, не соответствуют своей задаче - они не в состоянии дать нам ни знания, ни разумной веры, по крайней мере, в религиозных вопросах и в тех обстоятельствах, в которых мы находимся.

Для того чтобы продемонстрировать, что происходит, когда субъект, обладающий знанием на основании религиозного опыта, свидетельствует об этом другому субъекту, необходимо подробнее рассмотреть сам процесс передачи знания. Стоит заметить, что базовая функция передачи знания заключается в том, чтобы сохранить знание при передаче; мы не можем приписывать самому процессу необходимость проверки знания (Welbourne, 1979: 3). Поэтому надлежаще функционирующее свидетельство - это свидетельство, которое обеспечивает сохранение за убеждением статуса знания в процессе передачи ${ }^{10}$. Перед получателем знания также не стоит обязательной задачи проверить знание. Однако процесс передачи знания всё же предполагает предоставление получателю свидетельства соответствующего обоснования. В зависимости от того, что является источником обоснования, можно выделить два вида свидетельства.

В первом случае, который условно можно назвать передачей убеждения, агент $A$ передаёт знание агенту $B$, транслируя ему только пропозициональное содержание своего убеждения (далее-p). Например: «Число Пи приблизительно равно 3,14159» или «Самоубийство-грех». Когда $A$ сообщает $B$ о наличии у него некоторого убеждения, само это убеждение может уже присутствовать у $B$, однако убеждение, озвученное $A$, становится для $B$ обоснованным. При этом $B$ в данной ситуации так или иначе обосновывает вспомогательное убеждение типа

(1) Агент А обладает эпистемическим авторитетом ${ }^{11}$, хотя обоснование может отсутствовать в явной форме в зависимости от того, какому подходу к анализу природы свидетельства будет отдано предпочтение. Как только $A$ стал для $B$ авторитетом (во всех областях

10 Это работает несколько иначе в отношении свидетельских данных: на основании их передачи знание может возникнуть, а в случае передачи знания оно просто сохраняется. Однако в обоих случаях происходит передача опыта, поэтому указанное различие не имеет большого значения в данной статье.

${ }^{11} \mathrm{~B}$ данном случае понятие «эпистемический авторитет» используется для именования свойства субъекта, который заслуживает доверия. Дж. Адлер пишет об этом так: «Для слушающего доверять говорящему означает приписывать говорящему авторитет». (см. Adler, 2006) Какая-либо определенная концепция эпистемического авторитета в данном случае не подразумевается. 
знания или в каких-то определенных), для $B$ достаточно выполнения двух условий:

(2) $B$ знает, что $A$ знает, что $p$ (иначе: $A$ утверждает $B$, что $p$ ).

(3) $A$ является для $B$ авторитетом в области знаний, к которой относится убеждение $p$.

Условие (2) - это, собственно, само свидетельство. Если $B$ узнаёт от $A$, что ему известно, что $p$ (или $A$ утверждает, что р истинно), то при наличии доверия $B$ к $A$ у $B$ возникает основание для того, чтобы считать $p$ истинным. Хотя условие (3) очень важно, но, на мой взгляд, оно приобретается не в момент передачи знания, скорее, оно имплицитно содержится в (1). В момент передачи знания агент может перепроверить, распространяется ли на сообщаемое знание авторитет $A$, но вряд ли это можно считать обязательным условием. (3) может вообще не фигурировать, если речь идет о бытовых вопросах, не требующих какой-либо специализации. Поэтому я согласен с точкой зрения, что при наличии (1) для завершения передачи знания достаточно (2), т. е. того, что $B$ знает, что $A$ знает, что $p$ (Fricker, 2006: 595). В результате передачи убеждения агент $B$ обладает знанием, источником которого является свидетельство $A$. При передаче убеждения всегда имеется цепочка свидетельств, приводящая к самому первому субъекту, который обладает знанием, что $p$, не на основании свидетельства (ibid.: 6o6). Здесь я должен вспомнить про вторую разновидность сверхъестественных явлений, которую я назвал повторяющимися событиями. В качестве примера я рассматривал пресуществление в процессе таинства евхаристии. Как было упомянуто, знание о том, что данное явление сверхъестественно, не может быть получено из опыта, вызванного самим явлением. Это знание передаётся через свидетельство, и первым субъектом в этой цепочке является сам Бог. $\mathrm{K}$ проблеме человеческого опыта такого рода знание не имеет отношения. Именно поэтому передача такого рода знания не рассматривается подробно в данной статье ${ }^{12}$.

Способность сформировать знание на основании первого типа свидетельства обусловлена тем, что $B$ воспринимает $A$ как такого же эпистемического агента, как и он сам, поэтому он знает, что у $A$ может и должно быть обоснование для его знания, и поэтому он может приписывать ему авторитет. То же самое относится и к авторитетным

\footnotetext{
12 Данный вопрос целиком касается проблемы свидетельства и не имеет отношения к человеческому опыту. Главная сложность заключается в том, чтобы обосновать само существование свидетельства между Богом и первым человеком в цепочке авторитетов.
} 
письменным (аудио, визуальным) источникам, за которыми стоит один или несколько авторов: любой такой источник предполагает наличие субъекта, авторитет которого является обоснованием всех содержащихся в этом источнике знаний.

Особенностью этого вида свидетельства является то, что содержание свидетельства - при условии авторитетности агента $A$ - совершенно не влияет на обоснование его для $B$. Поэтому различия в содержании данного типа свидетельства - религиозном, научном, обыденном - не играют никакой роли в оценке его обоснованности. Наиболее яркий пример для науки - это работа в научном коллективе, состоящем из специалистов разных областей (Hardwig, 1991: 695-696). Вряд ли кто-то будет спорить, что наука как совокупность знаний формируется за счет надежной опытной основы. Однако для ученых в рамках одного сплоченного сообщества важна лишь возможность проверить предоставляемые данные, но в большинстве случаев свидетельство, полученное из научных статей, описаний экспериментов, отчетов коллег, не проверяется каждым субъектом в отдельности. Кроме того, этот вид свидетельства используется и при обучении, и при взаимодействии в обычной жизни. Практическое использование этого вида свидетельства не выявляет никаких отличий в передаче разных видов опыта. Необходимо заметить, что признанные большинством авторов критерии передачи знания соответствуют именно этому виду свидетельства, поскольку выглядят следующим образом: если $A$ знает, что $p, u A$ утверждает $B$, что $p$, и $B$ принимает р на основании свидетельства $A$, тогда $A$ знает, что $p$ (Adler, 2006). Формулировка «на основании свидетельства $A \gg$ прочно связывает данные критерии именно с передачей убеждения.

Второй вид свидетельства условно можно назвать передачей обоснования ${ }^{13}$. Это более редкий и весьма специфический способ свидетельства. В этом случае агент $A$ передаёт знание агенту $B$, пытаясь воспроизвести для агента $B$ доступное ему самому обоснование, чаще всего указывая ему на источник обоснования, а в наиболее простом случаепросто воспроизводя его. Так, агент $B$ воссоздает для агента $A$ условия, при которых убеждение, что $p$, станет обоснованным. Для этого вида

${ }^{13}$ Схожее, но не идентичное понимание передачи обоснования см. в Wright, 2015 . В статье передача обоснования - это слабая версия передачи знания, а не его разновидность. Кроме того, других разновидностей передачи знания автор в данном контексте не рассматривает. 
свидетельства очень важна связь между изначальным опытным обоснованием и агентом $B$, получающим знание. Агент $B$ должен получить рецепт обоснования передаваемого знания от агента $A$, и он должен иметь возможность им воспользоваться.

Отличие этого вида свидетельства - в том, что $B$ принимает , опираясь на обоснование, которое передаёт ему $A$, и обосновывающая сила складывается из свидетельства $A$ и внешнего обосновывающего фактора. Например, когда учитель химии говорит ученику: «Через 10 минут вода в этой колбе закипит, потому что установленная мной скорость нагрева составляет 10 градусов в минуту, а сейчас температура равняется нулю» - и ученик может проанализировать это высказывание; знание о том, что через 10 минут вода закипит, обосновано не только словами учителя, но и дополнительными внешними данными, которые получает сам ученик. Передача обоснования - это составной вид свидетельства, и его почти полностью можно редуцировать к иным источникам знания, чаще всего - к опыту и вышеуказанному свидетельству путем передачи убеждения. Однако я всё же считаю, что это самостоятельный вид передачи знания, поскольку нельзя редуцировать функцию учителя по передаче инструкций для получения опыта, что невозможно без наличия знания у $A$, ведь без наличия знания у $A$, агент $A$ не смог бы предоставить необходимые инструкции, а без инструкций агента $A$ агент $B$ не смог бы получить знание. Однако инструкции, полученные агентом $B$, мы не можем полностью отнести к свидетельству путем передачи убеждения ${ }^{14}$, поскольку в этой разновидности свидетельства агент $B$ вовсе не обязан доверять авторитету агента $A$ и главную роль в обосновании выполняет собственный опыт $B$. В случае передачи убеждения обосновывающую роль играет авторитет, установление особой связи между агентами необходимо.

Теперь можно выявить особенность второго типа свидетельствования в сочетании с религиозным опытом, сравнив ситуации, когда объект опыта является повторяющимся и когда он является эпистемически уникальным. Итак, передача обоснования всегда происходит через отсылку к повторяющимся и длящимся какое-то время явлениям. Предположим,

\footnotetext{
${ }^{14}$ Стоит заметить, что выделение данной разновидности свидетельства непринципиально для моей позиции и, скорее, создаёт дополнительные трудности для неё. Если признать, что этот способ свидетельства всё же можно редуцировать к сочетанию передачи убеждения и личному опыту, то мой итоговый тезис только усилится, поскольку это будет означать, что не существует самостоятельного вида свидетельства, несовместимого с передачей религиозного опыта.
} 
в момент времени $t_{1}$ агент А наблюдает некоторое явление $Z$. Затем в момент времени $t_{2}$ агент $A$ свидетельствует об этом событии агенту $B$ путем передачи обоснования. Если явление продолжается, агент $A$ может передать обоснование В в момент времени $t_{2}$, если явление $Z$ не продолжается, то агент $A$ не сможет передать обоснование в момент времени $t_{2}$. В случае с повторяющимися или длящимися какое-то время событиями можно рассчитывать, что когда-либо наступит такой момент, когда $Z$ снова повторится и обоснование можно будет передать. В случае с уникальными событиями такой момент не наступит никогда по определению. Таким образом, религиозный опыт, вызванный уникальным объектом, получен агентом $A$, и он имеет знание в момент времени $t_{1}$, но свидетельствовать об этом знании путем передачи обоснования в момент времени $t_{2}$ он не может.

Поэтому - несмотря на то что передача убеждения не выявляет никаких особенностей передачи религиозного знания - передача обоснования оказывается недоступна для религиозного знания, что легко объяснимо с точки зрения представленного выше подхода к пониманию уникальных сверхъестественных явлений. Специфика передачи обоснования требует, чтобы явление, с помощью которого мы обосновываем знание, было повторяющимся или длящимся какое-то время. Субъект $A$, как было сказано ранее, должен предоставить субъекту $B$ инструкцию, как получить обосновывающий опыт. Однако уникальное явление не позволяет представить такое обоснование, ведь оно по определению не может повториться. Это означает, что субъективность религиозного опыта возникает только тогда, когда субъект, получивший религиозный опыт, свидетельствует о нём путем передачи обоснования. Поэтому субъективность религиозного опыта локализована в одной разновидности свидетельства. Стоит заметить, что эта особенность свидетельствования проявляется в редких случаях, поскольку передача обоснования гораздо менее распространена на практике, чем передача убеждения. В изложенных рассуждениях показано, что данная особенность свидетельствования не может рассматриваться как сущностная особенность религиозного опыта. Причина её возникновения в том, что свидетельство в виде передачи обоснования оказывается несовместимо с уникальностью объекта религиозного опыта. Религиозный опыт субъекта, представляющего свидетельство, при возникновении этой особенности никак не изменяется и не приобретает новых свойств. 
ОШИБКИ В АНАЛИЗЕ СУБЪЕКТИВНОСТИ РЕЛИГИОЗНОГО ОПЫТА

Существует как минимум две возможные ошибки в анализе субъективности религиозного опыта. Основываясь на том, что явление уже не имеет место в $t_{2}$ и осуществить передачу обоснования невозможно, агент $B$ либо сам агент $A$ (в редких случаях) могут сделать один из двух ошибочных выводов: во-первых, что знание $A$ необоснованно, а во-вторых, что оно обладает свойством сильной субъективности.

Что касается первой ошибки, она состоит в следующем. Совершенно очевидно, что, после того как $A$ получил некоторый опыт, $B$ уже не сможет его получить, следовательно, блокируется возможность передачи обоснования. Отсюда можно сделать ошибочный вывод, что знание $A$ необоснованно. Олстон, например, говорит о том, что существует

путаница между убеждением кого-либо, что $p$ обоснованно, и тем, что некто имеет обоснование его убеждения, что $p$, где последнее предполагает, что он делает нечто, чтобы показать, что $p$, или чтобы показать, что его убеждение обосновано, или продемонстрировать его обоснование. [...] Удивительно, как часто путают эти ситуации в литературе (Alston, 1985: 58).

Вряд ли можно найти явные примеры такого утверждения в литературе, как об этом говорит Олстон, однако в неявной форме эта ошибка достаточно распространена. Утверждение, лежащее в основе этой ошибки, можно иначе сформулировать так: отсутствие у некоторого суббекта возможности обосновать убеждение означает необоснованность его убеждения.

Эта ошибка может произойти, например, в том случае, когда свидетельство путем передачи обоснования объявляется единственно возможным вариантом свидетельства, как это происходит в случае с верификационизмом. Допустим: $A$ увидел некое явление и обладает соответствующим опытом, достаточным для формирования знания, что это явление существует. $A$ хочет передать $B$ знание о том, что это явление существует. Допустим, что это явление эпистемически уникальное и ни $A$, ни $B$ не суждено увидеть его повторение. $A$ не может свидетельствовать об этом $B$ путем передачи обоснования. Всё что он может сделатьэто сообщить: «Я видел такое-то явление, факт того, что я его видел, подтверждает его существование». Для $B$ данный факт - это не обоснование. В качестве обоснования может служить только авторитет $A$. Но предположим, что $B$ не хочет использовать такой способ свидетельства, как передача убеждения, по какой-либо из причин (например, у него недостаточно обоснования, чтобы доверять $A$, или он полагает, что 
данный способ свидетельствования не является надежным). Получается, что ни один из видов свидетельства не доступен. Для $B$ знание не обосновано, потому что $A$ не может предоставить соответствующее обоснование. Однако отсюда нельзя делать вывод, что знание не обосновано и для $A$, поскольку мы не можем исключать возможность того, что $A$ стал свидетелем эпистемически уникального события. Если такой вывод всё же сделан - то это и есть пример первой ошибки ${ }^{15}$.

Вторая ошибка заключается в преувеличении степени субъективности религиозного опыта. До этого момента я не рассматривал различие между личным и публичным опытом, т. е. различие между внутренним опытом субъекта и опытом восприятия внешних явлений (Суинберн, Кедрова, 2014: 390-391). На мой взгляд, они не так уж важны, хотя существуют ситуации, в которых публичный опыт является более достоверным, и для дальнейших рассуждений это различие будет иметь значение. Ранее было проведено различие между невозможностью передачи опыта, или слабой субъективностью, и принципиальной невозможностью приобретения опыта другим субъектом, или сильной субъективностью. Затем я представил обоснование, что субъективность опыта локализована в одном из видов свидетельства. Однако какого рода субъективность присуща личному и публичному религиозному опыту? Довольно легко доказать, что опыт хоть и уникального, но публичного события обладает лишь слабой субъективностью. Действительно, если возвращаться к ситуации невозможности передачи обоснования от агента $A$, получившего опыт, к агенту $B$, то агент $B$ не может получить это знание на основании опыта не потому, что оно субъективно для $A$ или каким-то тесным образом связано с когнитивным аппаратом $A$, а потому, что оно уникально и уже произошло. При этом $B$ мог бы иметь опыт уникального события, если бы оказался в определенном месте в определенное время, поскольку оно, являясь публичным, было явлено всем очевидцам.

Более удивительным может показаться утверждение, что личный религиозный опыт также обладает свойством не сильной, а слабой субъективности. Представленная ранее линия рассуждений демонстрирует, что опыт восприятия уникальных объектов таков, что свидетельство

${ }^{15}$ В данном случае можно возразить: разве факт невозможности передачи знания не указывает на внутреннюю проблемную особенность опыта, вызываемого эпистемически уникальными событиями? Мой ответ: нет. Поскольку ДО того, как субъект $A$ соберется передать свой опыт, его опыт ничем не отличается от остальных. Проблема возникает лишь в момент передачи, что указывает на истинность тезиса (c). 
о нём не может быть осуществлено в форме передачи обоснования, однако может быть осуществлено в форме передачи убеждения. Причина этого: поскольку уникальное событие уже стало доступно для агента $A$ в прошлом, значит, оно никогда не будет доступно для агента $B$ в будущем. Это же с оговорками справедливо и для личного опыта. В случае если агент $A$ испытал некоторый опыт, который не может быть повторен, нечто вроде личного чуда (например, явление ангела во сне), то свидетельство об этом опыте путем передачи обоснования будет невозможно. Здесь, как может показаться, имеет место сильная форма субъективности, т. е. не только невозможность передачи обоснования от $A$ к $B$, но и невозможность для $B$ испытать этот опыт самостоятельно. В отличие от чуда, которое является публично, личный опыт происходит только с одним субъектом. И потому $B$ мог бы быть свидетелем чуда, но не мог бы стать свидетелем личного опыта $A$. Однако у нас нет оснований считать вышеописанную личную уникальность каким-то дополнительным свойством личного опыта, и поэтому нет оснований для приписывания этой ситуации сильной субъективности. Уникальный личный опыт мог бы иметь место и для $B$, но тогда он был бы недоступен для $A$. Причина, по которой он уже не произойдет для других субъектов, не в его внутреннем, личном характере, а опять же в том, что он уникален. Если личный религиозный опыт $A$ в этом случае субъективен, то он субъективен только исходя из сложившихся обстоятельств. Проще говоря - данный опыт мог быть уникально доступен для кого угодно (в том числе - для $B$ ), но обстоятельства сложились таким образом, что он стал уникально доступен для $A$. Если же речь идет не об уникальном личном опыте, а о повторяющемся, то достаточно сложно обосновать, почему его не может быть также и у $B$, если тот объект, который вызвал данный опыт у $A$, также может вызвать его и у $B$. Я не вижу возможностей для такого обоснования. Однако именно это требуется для доказательства утверждения о сильной субъективности религиозного опыта.

\section{ЗАКЛЮЧЕНИЕ}

Согласно представленному в первом разделе статьи подходу к анализу объектов религиозного и нерелигиозного опыта, отличие уникальных сверхъестественных от естественных явлений заключается в частоте их появления в опыте, однако иных отличий между данными явлениями опыта нет. Был также сделан вывод, что отличий между повторяющимися сверхъестественными явлениями и естественными явлениями 
в опыте нет, существующее отличие происходит не из опыта, а из свидетельства. Во втором разделе было показано, что различия в передаче религиозного и нерелигиозного опыта полностью объясняются особенностями свидетельства в виде передачи обоснования, что в совокупности с выводами первого раздела подтверждает тезисы (в), (с). Поскольку данный вид свидетельства используется достаточно редко, это не порождает больших проблем в обосновании религиозного знания. В третьем разделе было показано, что религиозный опыт-как личный, так и публичный - субъективен только в слабой форме, что подтверждает тезис (А). Представленная в статье модель объединения религиозного и нерелигиозного опыта может сработать для тех, кто разделяет мои взгляды на соотношение сверхъестественных и естественных объектов. Следующим шагом в разработке этой модели должна стать её универсализация. В частности, необходимо более детально рассмотреть все виды сверхъестественных явлений, постаравшись свести к нулю список возможных оговорок. Кроме того, моя модель не учитывает целевые и ценностные аспекты сверхъестественных явлений. В рамках данной концепции эти аспекты не имеют значения, однако для многих эти аспекты являются принципиальными. В то же время уже в текущем варианте мой подход демонстрирует, что объединение возможно, но пока что оно ограничено некоторыми мировоззренческими установками, несовместимыми с традиционным религиозным мировоззрением ${ }^{16}$.

\section{ЛИТЕРАТУРА}

Августин. О Граде Божием. Ххі // Творения. В 4 т. Т. 4 / под ред. С.И. Еремеева ; пер. с лат. [отцов Киевской духовной академии]. - СПб., Киев : Алетейя, УЦИММ-Пресс, 1998.

Греко Д. Свидетельство и передача религиозного знания / пер. с англ. К. В. Карпова // Эпистемология и философия науки. - 2017. - № 3. - С. 19-47. Джеймс У. Многообразие религиозного опыта : исследование человеческой природы / под ред. С. В. Лурье ; пер. с англ. В. Г. Малахиевой-Мирович, М. В. Шик. - М. : Академический проект, 2017.

Лейбнии Г. В. Рассуждение о метафизике / пер. с фр. Е. Н. Боброва // Сочинения. В 4 т. Т. 1 / под ред. В. В. Соколова. - М. : Мысль, 1982. - С. 125-163.

${ }^{16}$ Основой для данной статьи послужило выступление на семинаре «Знание и его границы: о чем следует молчать?», организованном Институтом философии РАН совместно с НИУ ВШЭ и Институтом христианской философии (Инсбрук, Австрия). Благодарю за ценные комментарии и вопросы участников семинара, а также анонимных рецензентов. 
Лейбнии, Г.В., Кларк С. Переписка с Кларком / пер. с фр., с англ. И.И. Свидерского, Г. Кребера // Сочинения. В 4 т. Т. 1 / Г. В. Лейбниц ; под ред. В. В. Соколова. - М. : Мысль, 1982. - С. 430-528.

Покорны П., Геккель У. Введение в Новый Завет / пер. с нем. В. М. Витковского. - М. : ББИ, 2019.

Прусс А. Р. Евхаристия : реальное присутствие и реальное отсутствие / пер. с англ. В.В. Васильева // Оксфордское руководство по философской теологии / под ред. Т.П. Флинта, М. К. Рея. - М. : Языки славянской культуры, 2013. - С. $74^{8-786 .}$

Суинберн Р. Существование Бога / пер. с англ. М. О. Кедровой. - М. : Языки славянской культуры, 2014 .

Фома Аквинский. Сумма теологии. Часть І. Вопросы $75^{-119}$ / пер. с лат. С. И. Еремеева. - Киев : Ника-Центр, 2005.

Adler J. Epistemological Problems of Testimony / The Stanford Encyclopedia of Philosophy ; ed. by E. N. Zalta. - 2006. - URL: https://plato.stanford.edu /entries/testimony-episprob/ (visited on Sept. 3, 2019).

Alston W. Concepts of Epistemic Justification // The Monist. - 1985. - Vol. 68. P. $57^{-89}$.

Clarke $S$. From a Discourse Concerning the Unchangeable Obligations of Natural Religion // Samuel Clarke : A Demonstration of the Being and Attributes of God. And Other Writings / ed. by E. Vailati. - Cambridge : Cambridge University Press, 1998. - P. $147^{-1} 5^{0}$.

Fricker E. Second-Hand Knowledge // Philosophy and Phenomenological Research. 2006. - Vol. 73, no. 3. - P. 592-618.

Hardwig J. The Role of Trust in Knowledge // The Journal of Philosophy. - 1991. Vol. 88, no. 12. - P. 693-708.

Hume D. An Enquiry Concerning Human Understanding // Hume : An Enquiry Concerning Human Understanding and Other Writings / ed. by S. Buckle. Cambridge : Cambridge University Press, 2007. - P. 1-131.

Rowley W. D. Evidence of Evidence and Testimonial Reductionism // Episteme. 2012. - Vol. 9, no. 4. - P. 377-391.

Welbourne M. The Transmission of Knowledge // The Philosophical Quarterly. 1979. - Vol. 29, no. 114. - P. 1-9.

Wright S. The Transmission of Knowledge and Justification / Synthese. - 2015. URL: http://www.stephenwrightphilosophy.com/uploads/3/4/6/2/34628466/the_ transmission_of_knowledge_and_justification_website_version.pdf (visited on Dec. 3, 2019). 
Sysoyev, M. S. 2020. "Religioznyy opyt i svidetel'stvo [Religious Experience and Testimony]" [in Russian]. Filosofiya. Zhurnal Vysshey shkoly ekonomiki [Philosophy. Journal of the Higher School of Economics] 4 (4), 50-76.

\title{
MATVEY SYSOYEV
}

TEACHING Assistant

N. N. Burdenko Voronezh State Medical University (Voronezh, Russia); ORCID: 0000-0003-1152-577X

\section{Religious EXPERIENCE AND TESTIMONY}

Submitted: Jan. 28, 2020. Reviewed: Apr. 20, 2020. Accepted: Jan. 12, 2020.

\begin{abstract}
The paper considers the problem of the transfer of religious experience, consisting of two elements: the problem of the features of individual religious experience and the problem of testimony in relation to religious experience. Religious experience seems to be more difficult to transfer through testimony than other types of experience. The search for the causes of these features leads either to the assertion that these are features of the religious experience itself, or to the assertion that these are features of a certain type of testimony. Based on these features and, most often, adopting the first option, opponents of theism try to reduce religious experience to cognitive distortions and disorders, and supporters of theism try to create a special epistemological description of this experience in order to protect it. The author made an attempt to substantiate the thesis that difficulties in transferring religious experience arise in special specific cases and only due to the features of the transfer of experience, and not due to the features of religious experience as such. These statements are based on the approach proposed by the author to the analysis of objects of religious, scientific, and everyday experience. This approach combines natural and supernatural objects of experience into a single class of objects. The most significant feature of the approach is the assertion that such unique supernatural objects of experience as miracles differ from other objects of experience only epistemically, but not ontologically. Considering the epistemological consequences of this approach, the author comes to the stated theses.
\end{abstract}

Keywords: Epistemology of Religion, Theism, Religious Experience, Supernatural, Testimony, Transmission of Knowledge.

DOI: $10.17323 / 2587-8719-2020-4-50-76$.

\section{REFERENCES}

Adler, J. 2006. "Epistemological Problems of Testimony." The Stanford Encyclopedia of Philosophy. Accessed Sept. 3, 2019. https://plato.stanford.edu/entries/testimony-episprob/. Alston, W. 1985. "Concepts of Epistemic Justification." The Monist 68:57-89.

Augustinus. 1998. "O Grade Bozhiyem. XXI [De Civitate Dei XxI]" [in Russian]. In vol. 4 of Tvoreniya [Selected Works], ed. by S. I. Yeremeyev, trans. from the Latin by [Priests of Kiev Theological Academy]. 4 vols. Sankt-Peterburg [Saint Petersburg] and Kiyev [Kiev]: Aleteyya / UTsIMM-Press.

Clarke, S. 1998. "From a Discourse Concerning the Unchangeable Obligations of Natural Religion." In Samuel Clarke: A Demonstration of the Being and Attributes of God. And Other Writings, ed. by E. Vailati, 147-150. Cambridge: Cambridge University Press.

Fricker, E. 2006. "Second-Hand Knowledge." Philosophy and Phenomenological Research 73 (3): $592-618$. 
Greco, J. 2017. "Svidetel'stvo i peredacha religioznogo znaniya [Testimony and the Transmission of Religious Knowledge]" [in Russian], trans. from the English by K. V. Karpova. Epistemologiya i filosofiya nauki [Epistemology \& Philosophy of Science], no. 3: 19-47.

Hardwig, J. 1991. "The Role of Trust in Knowledge." The Journal of Philosophy 88 (12): $693-708$.

Hume, D. 2007. "An Enquiry Concerning Human Understanding." In Hume: An Enquiry Concerning Human Understanding and Other Writings, ed. by S. Buckle, 1-131. Cambridge: Cambridge University Press.

James, W. 2017. Mnogoobraziye religioznogo opyta [The Varieties of Religious Experience]: issledovaniye chelovecheskoy prirody [A Study in Human Nature] [in Russian]. Ed. by S. V. Lur'ye. Trans. from the English by V. G. Malakhiyeva-Mirovich and M. V. Shik. Moskva [Moscow]: Akademicheskiy proyekt.

Leibniz, G.W. 1982. Rassuzhdeniye o metafizike [Discours de métaphysique] [in Russian]. In vol. 1 of Sochineniya [Sollected Works], ed. by V. V. Sokolov, trans. from the French by Ye. N. Bobrov, 125-163. 4 vols. Moskva [Moscow]: Mysl'.

Leibniz, G. W., and S. Clarke. 1982. Perepiska s Klarkom [Leibniz-Clarke Correspondence] [in Russian]. In vol. 1 of Sochineniya [Sollected Works], by G. W. Leibniz, ed. by V. V. Sokolov, trans. from the French and from the English by I. I. Sviderskiy and G. Kreber, 430-528. 4 vols. Moskva [Moscow]: Mysl'.

Pokorny, P., and U. Heckel. 2019. Vvedeniye v Novyy Zavet [Einleitung in das Neue Testament] [in Russian]. Trans. from the German by V. M. Vitkovskiy. Moskva [Moscow]: BBI.

Pruss, A. R. 2013. "Yevkharistiya [The Eucharist]: real'noye prisut-stviye i real'noye ot-sut-stviye [Real Presence and Real Absence]" [in Russian]. In Oksfordskoye rukovodstvo po filosofskoy teologii, ed. by T. P. Flint and M. K. Rey, trans. from the English by V. V. Vasil'yev, 748-786. Moskva [Moscow]: Yazyki slavyanskoy kul'tury.

Rowley, W. D. 2012. "Evidence of Evidence and Testimonial Reductionism." Episteme 9 (4): 377-391.

Swinburne, R. 2014. Sushchestvovaniye Boga [The Existence of God] [in Russian]. Trans. from the English by M. O. Kedrova. Moskva [Moscow]: Yazyki slavyanskoy kul'tury.

Thomas Aquinas. 2005. Summa teologii. Chast' I. Voprosy 75-119 [Summa Theologiae Ia] [in Russian]. Trans. from the Latin by S. I. Yeremeyev. Kiyev [Kiev]: Nika-Tsentr.

Welbourne, M. 1979. "The Transmission of Knowledge." The Philosophical Quarterly 29 (114): $1-9$.

Wright, S. 2015. "The Transmission of Knowledge and Justification." Synthese. Accessed Dec. 3, 2019. http://www. stephenwrightphilosophy.com/uploads/3/4/6/2/34628466/the_tr ansmission_of_knowledge_and_justification_website_version.pdf. 